\title{
LOS CONOCIMIENTOS SOBRE RASGOS DE PERSONALIDAD DEL PROFESORADO COMO FACILITADORES DE LA INNOVACIÓN EDUCATIVA: ESTADO DEL ARTE1
}

\section{KNOWLEDGE ON PERSONALITY TRAITS OF TEACHERS AS FACILITATOR OF EDUCATIONAL INNOVATION: THE STATE OF THE ART}

\author{
Carlos Monge López \\ David Montalvo Saborido \\ Patricia Gómez Hernández \\ Universidad de Alcalá
}

Fecha de recepción: 25/10/2013

Fecha de aceptación: 12/04/2014

\section{RESUMEN}

La innovación educativa es un conjunto de procesos que tienen como objetivo principal la mejora de los procesos de enseñanza-aprendizaje. Así, los centros educativos procuran dirigir acciones de cambio orientadas a la mejora y la calidad. Sin embargo, generalmente esto no resulta una tarea sencilla, sino que existen diversas barreras que dificultan la innovación educativa, aunque también hay facilitadores de la misma. Por ende, haciendo referencia a estos últimos, la clave puede estar en determinar cuáles son y cómo manejarlos. Concretamente, son diversos los autores que apuestan por una serie de elementos encaminados a mejorar los procesos innovadores dentro de los centros educativos. Sin embargo, uno de esos elementos y que la literatura científica no aborda de manera sistemática y relevante es cómo los rasgos de personalidad influyen en las prácticas docentes y organizativas innovadoras. Por ello, lo que aquí se pretende es mostrar el estado del arte sobre cómo los conocimientos acerca de los rasgos de personalidad pueden actuar como facilitadores de la innovación educativa. De esta manera, si un miembro de la comunidad educativa (ya sea docente, asesor, etc.) conoce cómo son sus compañeros y él mismo, en cuanto a características personales se refiere, probablemente se favorezca la gestión de ciertos procesos de cambio dirigidos a la mejora educativa. Concretamente, dentro de esas características individuales más estudiadas se encuentran los rasgos de personalidad, aunque también se incluyen aspectos como el autoconcepto, los procesos atributivos, la estima, etc.

Palabras clave: características personales, estado del arte, facilitadores del cambio, innovación pedagógica, rasgos de personalidad, resistencia al cambio.

\begin{abstract}
The educational innovation is a set of processes whose main is to aim improve the teaching-learning process. Thus, schools seek to change actions directed to improve and give quality. However, this is generally not an easy task, but there are several

\footnotetext{
1 Estudio realizado gracias a la Beca de Iniciación en la Actividad Investigadora del Programa Propio de la Universidad de Alcalá
} 
Los conocimientos sobre rasgos de personalidad del profesorado como facilitadores de la innovación educativa: estado del arte

barriers to educational innovation, although there are facilitators as well. Therefore, referring to the latter, the key may be located in determining what and how to handle them. Specifically, some authors advocate a number of elements in order to improve innovation processes within schools. But despite that, the scientific literature does not address systematically the influence of personality traits and organizational practices in innovative teachers. So, the main aim of this paper is to show the state of the art about how knowledge about personality traits may act as facilitators of educational innovation. Thus, if a member of the educational community (whether teacher, counselor, etc.) knows what their peers and himself, as to personal characteristics are concerned, probably favoring the management of certain processes of change aimed at educational improvement. Specifically, within these individual characteristics, personality traits are the most studied, but they also include aspects such as selfconcept, attributional processes, esteem, etc.

Key words: educational innovation, individual characteristics, facilitators to change, personality traits, state of the art, resistance to change.

\section{INTRODUCCIÓN}

El conjunto de instituciones educativas busca mejorar los procesos de enseñanza-aprendizaje, lo que, como se muestra más adelante, está estrechamente relacionado con el término innovación. Por ello, lo que aquí se pretende es mostrar el estado de la cuestión sobre cómo ciertos elementos pueden ejercer la función de facilitadores en los procesos de innovación educativa. Así, tras un análisis que aborda los potenciadores y restrictores sobre la temática, se presenta un aspecto escasamente tratado y de manera poco sistemática en la literatura científica en relación a la innovación educativa: los rasgos de personalidad.

La personalidad puede estar constituida por diversos elementos. Así, entre otros aspectos, pueden incluirse (Bermúdez, Pérez-García, Ruiz, Sanjuán y Rueda, 2011): los rasgos como estructura estable de la personalidad, la motivación dentro del sistema de características individuales, la autorregulación conductual, la identidad personal, el locus de control, etc. Sin embargo, de todos ellos, posiblemente, los rasgos de personalidad son, como se señala a continuación, unos de los más relevantes.

Concretamente, hay una evidencia empírica que demuestra la relación existente entre algunos rasgos de personalidad y la innovación educativa, prestando especial atención a las características individuales del profesorado (Harvey, 19672; Lazarsfield y Katz, 19632; Miles, 19642; Ríos, 2004, 2006a, 2006b y 2009). Sin embargo, todavía no se ha demostrado de manera significativa cómo los conocimientos sobre esta temática puedan actuar como facilitadores de las mismas. Por ello,

2 Citado/s en Huberman (1973). 
aquí se plantea una aproximación teórica que defiende esta postura, con la finalidad última de que sus aportaciones y potencialidades contribuyan a crear, planificar y poner en práctica innovaciones que mejoren los procesos educativos dentro de los centros.

En este sentido, el conocer las características individuales de los compañeros puede mejorar los procesos de influencia personal, tanto para desarrollar la cohesión grupal como para intentar que los demás hagan lo que uno pretende hacer. Así, los gestores de las innovaciones educativas si conocen cómo piensan sus compañeros, sus motivaciones, las formas de comportarse, etc. y, en definitiva, su personalidad. De esta manera, estos gestores pueden ejercer una influencia para buscar la conexión del grupo y, así, facilitar los procesos innovadores en los centros educativos. Sin embargo, la personalidad es un constructo muy amplio y difícil de abarcar por una única persona, especialmente si tiene que conocer la propia de un conjunto extenso de personas. Por tanto, los rasgos de personalidad (que reducen el conjunto de variables teóricas agrupándolas en factores) pueden minimizar el esfuerzo cognitivo que realizan las personas para conocer cómo es el comportamiento de los demás.

Por otro lado, posiblemente cualquier persona con conocimientos sobre el ámbito educativo conozca diferentes perfiles de sus formadores. Sin embargo, la mayoría de ellos son recordados principalmente debido a sus peculiaridades, inquietudes, modos de hablar y vestir, etc.; en síntesis: por su personalidad.

En la actualidad, se puede afirmar que la educación avanza de manera más lenta de lo que lo hace la sociedad. Para ello, y con el fin de ofrecer todas las ventajas posibles para conseguir una educación del siglo XXI se precisa innovar (Imbernón, 2006). En algunos casos, la innovación en educación se asocia con ciertos recursos tecnológicos (p. e: pizarras digitales, ordenadores, tabletsy demás), que en numerosas ocasiones se relacionan con las nuevas metodologías (p. e.: m-leraning, plataformas virtuales, etc.). Sin embargo, las innovaciones no dependen ni de los recursos metodológicos ni de los materiales (aunque sí ayudan), sino que verdaderamente se crean y mantienen gracias a los participantes más directos en los procesos de enseñanzaaprendizaje.

Por todo ello, aquí se realiza un análisis sobre qué son los rasgos de personalidad, entendidos como constructos estructurales de la misma, así como cuáles y en qué consisten las principales teorías al respecto. Seguidamente se presenta una conceptualización sobre innovación educativa, sus tipologías y las variables más relevantes que influyen 
sobre ella, ya sea para facilitar su implantación y gestión como para restringirla. Posteriormente se señalan algunos estudios sobre la relación entre las características personales e innovación en el ámbito educativo, enfatizando algunos de los datos empíricos e hipótesis teóricas mediante las cuales se pretende responder a la cuestión sobre qué rasgos de personalidad poseen los docentes que más innovan en su práctica educativa. Finalmente, se concluye que el conocer cómo son los miembros de los centros educativos (especialmente los docentes, directivos y equipos psicopedagógicos), en lo que a rasgos de personalidad se refiere, puede ayudar a gestionar las innovaciones, facilitando que los participantes se impliquen.

\section{LOS RASGOS DE PERSONALIDAD}

\subsection{Conceptualización}

Los rasgos de personalidad suelen ser entendidos como los elementos estables que constituyen la estructura interna de la personalidad. Sin embargo, todavía no existe una definición universalmente aceptada al respecto. Por este motivo, se debe partir de la conceptualización de personalidad.

Por ejemplo, tras un análisis de diversas definiciones, Pérez-García y Bermúdez (2011) señalan que:

La personalidad hace referencia a la forma de pensar, percibir o sentir de un individuo, que constituye su auténtica identidad, y que está integrada por elementos de carácter más estable (rasgos) y elementos cognitivos, motivacionales y afectivos más vinculados con la situación y las influencias socio-culturales, y, por tanto, más cambiables y adaptables a las peculiares características del entorno, que determinan, en una continua interrelación e interdependencia, la conducta del individuo, tanto lo que podemos observar desde fuera (conducta manifiesta), como los nuevos productos cognitivos, motivacionales o afectivos (conducta privada o interna), que entrarán en juego en la determinación de la conducta futura (cambios en expectativas, creencias, metas, estrategias, valoración de las situaciones, etc.) (p. 34).

Dependiendo de la teoría sobre los rasgos de personalidad que se aborde, éstos se pueden definir de una manera u otra. Pero, a pesar de eso, existen posturas conciliadoras que intentan aportar una conceptualización más generalizada. De esta manera, los rasgos de 
personalidad se pueden entender como la consistencia de la respuesta de un individuo ante distintas situaciones y se aproxima al concepto que la gente utiliza para describir la conducta de los demás, es decir, los rasgos son constructos teóricos que se utilizan para describir a las personas y comparar unas con otras (Pérez-García y Bermúdez, 2011). Precisamente, de ahí se pueden obtener las características principales. Así, otros autores (Ruiz, 2007) señalan como particularidades de los rasgos que son disposiciones internas, estables y consistentes, no observables directamente, que influyen en el modo de comportamiento de las personas y en las diferencias que se observan entre ellas.

Respecto a los rasgos de personalidad existen multitud de argumentos a favor y en contra. En este sentido, Montalvo (2012) recoge varias críticas y defensas. En primer lugar, entre algunas de las conclusiones a favor de la teoría de los rasgos destacan las aportadas por Eysenck y Eysenck (1980)3; que pueden resumirse en las siguientes:

- Las personas se distinguen en importantes disposiciones relativamente permanentes y genéticas de la personalidad que se conocen como rasgos.

- Los rasgos están en constante interacción con el medio donde se desenvuelve el sujeto, produciendo ciertas condiciones transitorias internas denominadas estados.

- Los rasgos y los estados son variables intermedias o mediadoras, útiles para explicar las diferencias individuales en la conducta, existiendo una relación entre ambos indirecta, ya que se encuentra modulada por otros factores situacionales o del entorno.

Por otro lado, Montalvo (2012) continúa señalando algunas de las críticas, siendo las más destacadas las realizadas por Mischel (1968)4. Fundamentalmente, éstas se dirigen hacia cuestiones relativas a los datos estadísticos. Así, inicialmente, puede considerarse que tanto las muestras utilizadas como los instrumentos presentan limitaciones y poca fiabilidad. Entonces, Eysenck (1985 y 1987) ${ }^{4}$ como contraargumento a las críticas de Mischel (1968) ${ }^{4}$ propone una serie de explicaciones al respecto, confirmando la utilización del análisis factorial como herramienta para hallar el porcentaje de variabilidad que explica un factor sobre un determinado rasgo. Concretamente, por análisis factorial se puede entender al "método multivariante que intenta explicar, mediante un modelo lineal, un conjunto amplio de variables o individuos según un número reducido de variables hipotéticas llamadas factores" (Gil Pascual, 2011, p. 61). En definitiva, se trata de agrupar un

${ }^{3}$ Citados en Montalvo (2012).

${ }^{4}$ Citados en Montalvo (2012). 
Los conocimientos sobre rasgos de personalidad del profesorado como facilitadores de la innovación educativa: estado del arte

número de variables obteniendo una cantidad más reducida de factores que explican la covarianza existente entre las variables.

En síntesis, la estructura de la personalidad está compuesta por unas disposiciones estables denominadas rasgos, que ejercen influencia sobre la conducta de las personas, que sirven para categorizarlas y que no se pueden observar directamente. Por ende, el procedimiento generalmente utilizado para determinar los rasgos de personalidad es el análisis factorial de los datos obtenidos en cuestionarios.

\subsection{Principales teorías}

La personalidad es objeto de estudio desde tiempos de la Grecia clásica. Por ejemplo, Empédocles e Hipócrates en el siglo $\vee$ a. C. postulan la teoría de los humores (ver Tabla 1). La idea básica es que las personas son una parte del universo que les rodea $y$, por consiguiente, deben estar constituidas por todos los elementos que lo componen (aire, tierra, fuego y agua). Además, cada elemento se corresponde con un fluido corporal (sangre, flema, bilis amarilla y bilis negra), que, debido a sus propiedades, da lugar a un temperamento que, a su vez, se encuentra caracterizado por un atributo predominante (optimismo, tristeza, ira y apatía).

\begin{tabular}{|c|c|c|c|c|}
\hline Elementos & Propiedades & Humor & Temperamento & Predominancia \\
\hline Aire & $\begin{array}{l}\text { Cálido y } \\
\text { húmedo }\end{array}$ & Sangre & Sanguíneo & Optimismo \\
\hline Tierra & Frío y seco & Bilis negra & Melancólico & Tristeza \\
\hline Fuego & $\begin{array}{l}\text { Cálido y } \\
\text { seco }\end{array}$ & $\begin{array}{l}\text { Bilis } \\
\text { amarilla }\end{array}$ & Colérico & Ira \\
\hline Agua & $\begin{array}{l}\text { Frío y } \\
\text { húmedo }\end{array}$ & Flema & Flemático & Apatía \\
\hline
\end{tabular}

Tabla $\mathrm{n}^{\circ} 1$. Teoría de los humores (Pelechano y Servando, 2004)

Estos postulados son la base de numerosas teorías posteriores y más recientes. Así, a finales del siglo XIX, la propuesta de Wundt modifica la tipología hipocrática y la hace depender de dos bases biológicas (ver Tabla 2). Concretamente, combina la velocidad de excitación emocional (rápida y lenta) con la intensidad de reacción (fuerte y débil).

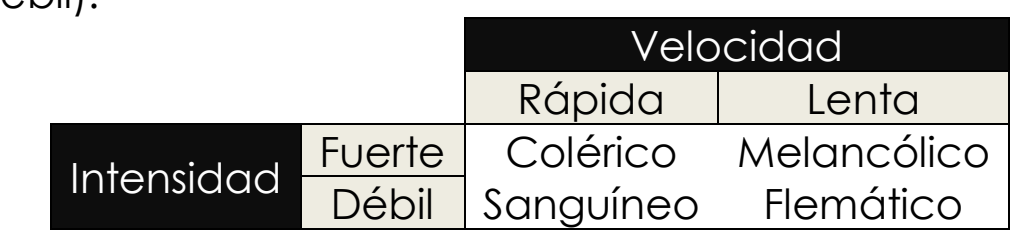

Tabla ${ }^{\circ} 2$. Propuesta de Wundt (Pelechano y Servando, 2004) 
También, ya en el primer tercio del siglo XX, según Pavlov existe una diferencia entre los organismos con sistema nervioso débil (melancólicos) y organismos con el sistema nervioso fuerte, que pueden ser no equilibrados (sanguíneos) o equilibrados, divididos, a su vez, en impulsivos (coléricos) y lentos (flemáticos).

A pesar de todo ello, es posible que los estudios de Heymans y Wiersma en 1909 sean los comienzos de la investigación moderna sobre este ámbito (Montalvo, 2012). Precisamente, son ellos los que empiezan a utilizar el análisis factorial sistemáticamente para determinar los rasgos de personalidad, que son tres: intensidad emocional, impulso general y, finalmente, extraversión-introversión.

Efectivamente, son diversas las teorías sobre rasgos de personalidad que perduran en la actualidad. Entre ellas se pueden encontrar la propuesta por Cattell (1949), el modelo de los cinco factores (Caprara, Barbaranelli y Borgogni, 1993; Costa y McCrae, 1978), el de Eysenck (1975), el de Gray (1981), la teoría de Guilford (1959), el modelo heptafactorial (Zuckerman, 2002), etc.

En primer lugar, según la teoría de Cattell, existen diversos tipos de rasgos, y pueden ser clasificados atendiendo a varios criterios (Ruiz, 2007):

- Rasgos comunes (aquellos que están presentes en todas las personas) y rasgos únicos (aquellos que solamente se observan en una persona y cuya manifestación no es igual en ningún otro individuo).

- Rasgos superficiales (son características de personalidad asociadas a un conjunto de rasgos que suelen aparecer juntos en distintas ocasiones) y rasgos fuentes (son entidades causales responsables de la conducta que se manifiesta a través de los rasgos superficiales). Además, los rasgos fuentes, a su vez, pueden ser generales (aquellos que afectan a la conducta en muchas ocasiones diferentes) o específicos (son los que atañen a la conducta en situaciones muy concretas).

- Rasgos motivacionales (aquellos que están implicados en las acciones para alcanzar una determinada meta), rasgos aptitudinales (son los que están relacionados con la eficiencia para alcanzar dicha meta) y rasgos temperamentales (aquellos que tratan sobre el estilo de respuesta).

También, considera que existen tres tipos de fuentes que aportan información sobre los rasgos de personalidad (Montalvo, 2012): 
Los conocimientos sobre rasgos de personalidad del profesorado como facilitadores de la innovación educativa: estado del arte

- Live (L): es aquella información referida a conductas en situaciones reales; registros de vida.

- Questionnaire (Q): es la información obtenida a partir de autoinformes.

- Objetive Tests (OT): es la información obtenida a partir de pruebas objetivas, donde el sujeto no es consciente entre la respuesta y lo que se evalúa.

En sus investigaciones iniciales, Cattell y sus colaboradores (Cattell, Cattell y Cattell, 1949) procuran determinar cómo se relacionan los datos generados a partir de las diversas fuentes. Para ello realiza diversos análisis léxicos y factoriales, lo que propicia la creación de un cuestionario estandarizado (16 Personality Factor, 16PF) revisado en diversas ocasiones y adaptado a varias lenguas y culturas. En él, Cattell determina que son 16 las escalas primarias de personalidad: afabilidad, razonamiento, estabilidad, dominancia, animación, atención a normas, atrevimiento, sensibilidad, vigilancia, abstracción, privacidad, aprensión, apertura al cambio, autosuficiencia, perfeccionismo y tensión.

En segundo lugar, a partir de la creación de diversos cuestionarios y la factorización con otros grados de rotación de los ya existentes (como el 16PF), se encuentra el modelo de los cinco factores. Sin embargo, no es una perspectiva única (como, por ejemplo, la de Cattell), sino que existen diversas aproximaciones a este modelo (ver Tabla 3). Pero los autores más destacados son, por un lado, Costa y McCrae (1978) y, por otra parte, Caprara y otros (1993).

\begin{tabular}{|c|c|c|c|c|c|}
\hline & Factor I & Factor II & Factor III & Factor IV & Factor V \\
\hline Fiske (1949) & Confianza & $\begin{array}{l}\text { Adaptabilid } \\
\text { ad social }\end{array}$ & Conformidad & $\begin{array}{l}\text { Control } \\
\text { emocional }\end{array}$ & $\begin{array}{l}\text { Intelecto } \\
\text { crítico }\end{array}$ \\
\hline $\begin{array}{l}\text { Norman } \\
\text { (1963) }\end{array}$ & Surgencia & Amabilidad & $\begin{array}{l}\text { Responsabilid } \\
\text { ad }\end{array}$ & $\begin{array}{l}\text { Estabilidad } \\
\text { emocional }\end{array}$ & Cultura \\
\hline $\begin{array}{l}\text { Borgatta } \\
\text { (1964) }\end{array}$ & $\begin{array}{l}\text { Asertivida } \\
\mathrm{d}\end{array}$ & Amabilidad & $\begin{array}{l}\text { Interés por la } \\
\text { tarea }\end{array}$ & $\begin{array}{l}\text { Emocionalid } \\
\text { ad }\end{array}$ & $\begin{array}{l}\text { Inteligenc } \\
\text { ia }\end{array}$ \\
\hline $\begin{array}{l}\text { Digman y } \\
\text { Takemoto } \\
\text { (1981) }\end{array}$ & $\begin{array}{l}\text { Extraversió } \\
\mathrm{n}\end{array}$ & Amistad & $\begin{array}{l}\text { Tendencia al } \\
\text { logro }\end{array}$ & Ansiedad & Intelecto \\
\hline $\begin{array}{l}\text { Goldberg } \\
\text { (1981) }\end{array}$ & Surgencia & Amabilidad & $\begin{array}{l}\text { Responsabilid } \\
\text { ad }\end{array}$ & $\begin{array}{l}\text { Estabilidad } \\
\text { emocional }\end{array}$ & Intelecto \\
\hline $\begin{array}{l}\text { Conley } \\
\text { (1985) }\end{array}$ & $\begin{array}{l}\text { Extraversió } \\
\text { n social }\end{array}$ & Amabilidad & $\begin{array}{l}\text { Control del } \\
\text { impulso }\end{array}$ & $\begin{array}{l}\text { Neuroticism } \\
\text { o }\end{array}$ & $\begin{array}{l}\text { Interés } \\
\text { intelectu } \\
\text { al }\end{array}$ \\
\hline $\begin{array}{l}\text { Raad, } \\
\text { Mulder, } \\
\text { Kloosterma } \\
\text { n (1988) }\end{array}$ & $\begin{array}{l}\text { Extraversió } \\
\mathrm{n}\end{array}$ & Amabilidad & $\begin{array}{l}\text { Responsabilid } \\
\text { ad }\end{array}$ & $\begin{array}{l}\text { Inestabilida } \\
\text { d } \\
\text { emocional }\end{array}$ & Cultura \\
\hline
\end{tabular}




\begin{tabular}{|c|c|c|c|c|c|}
\hline $\begin{array}{l}\text { Botwin y } \\
\text { Buss (1989) }\end{array}$ & $\begin{array}{l}\text { Extraversió } \\
\mathrm{n}\end{array}$ & Amabilidad & $\begin{array}{l}\text { Responsabilid } \\
\text { ad }\end{array}$ & Seguridad & Cultura. \\
\hline $\begin{array}{l}\text { Peabody y } \\
\text { Goldberg } \\
\text { (1989) }\end{array}$ & Poder & Amor & Trabajo & Afecto & Intelecto \\
\hline $\begin{array}{l}\text { Tupes } \\
\text { Christal } \\
\text { (1992) }\end{array}$ & Surgencia & Amabilidad & $\begin{array}{l}\text { Dependenci } \\
\text { a }\end{array}$ & $\begin{array}{l}\text { Estabilidad } \\
\text { emocional }\end{array}$ & Cultura \\
\hline
\end{tabular}

Tabla $n^{\circ} 3$. Algunos modelos de cinco factores (adaptado de Escrivá, 2009)

Costa y McCrae (1978), tras un análisis y diferentes rotaciones factoriales de diversos cuestionarios de personalidad, elaboran el suyo propio (Neuroticims, Extraversion and Openness-Personality Inventory, NEO-PI). Y con él determinan que son cinco los rasgos de personalidad (extraversión, amabilidad, responsabilidad, neuroticismo y apertura a la experiencia), de los cuales cada uno posee varias facetas.

Caprara y otros (1993) también crean su propio cuestionario (Big Five Questionnaire, BFQ), donde señalan que la personalidad está constituida fundamentalmente por cinco factores, que a su vez están compuestos por dos subdivisiones cada uno: energía (dinamismo y dominancia), afabilidad (cooperación y cordialidad), tensión (escrupulosidad y perseverancia), estabilidad emocional (control de las emociones y control de los impulsos) y apertura mental (apertura a la cultura y apretura a la experiencia).

También Zuckerman (2002) propone una modelo pentafactorial. Precisamente, después de diversas investigaciones sobre cuántos y cuáles son los principales rasgos de personalidad, sostiene que ésta está formada fundamentalmente por cinco factores: (1) búsqueda impulsiva de sensaciones, (2) neuroticismo-ansiedad, (3) agresión-hostilidad, (4) actividad y (5) sociabilidad.

En tercer lugar, Eysenck y Eysenck (1975) fundamentan su teoría en el modelo explicativo de Pavlov y el modelo bidimensional de Wundt, lo que, a diferencia de los autores anteriores, les lleva a postular que la personalidad se encuentra prácticamente determinada por variables hereditarias (Montalvo, 2012). Así, tras varios análisis factoriales, estos autores postulan que existen tres rasgos de segundo orden (suprafactores) compuestos por varios factores de primer orden (Ruiz, 2007):

- Extraversión-introversión: sociabilidad, despreocupación, surgencia, vitalidad, actividad, dominancia, aventura, búsqueda de sensaciones y dogmatismo. 
- Neuroticismo-estabilidad emocional: ansiedad, desestimación personal, timidez, depresión, emotividad, tensión, irracionalidad, culpabilidad y tristeza.

- Psicoticismo-control de los impulsos: agresividad, antisociabilidad, apatía, frialdad, creatividad, egocentrismo, impersonalidad, inconmovilidad e impulsividad.

En cuarto lugar, Gray (1981) realiza un estudio donde, primeramente, aplica el cuestionario de Eysenck a una muestra de sujetos y, seguidamente, en el análisis factorial rota los datos obtenidos de manera diferente a los anteriores estudios. De esta manera, encuentra dos dimensiones fundamentales de la personalidad: ansiedad e impulsividad. Concretamente, la primera se corresponde con valores altos de neuroticismo e introversión, mientras que la segunda parte de puntuaciones altas en neuroticismo y extraversión.

En quinto lugar, Guilford y sus colaboradores (Guilford, 1959) diseñan diversos cuestionarios psicométricos para la evaluación de la personalidad, en los cuales se miden rasgos diferentes. Sin embargo, en todos ellos aparecen tres rasgos como especialmente replicables (Montalvo, 2012): (a) actividad social, (b) introversión-extraversión y (c) madurez emocional-neuroticismo; a los cuales añade (d) disposición paranoide. Pero, finalmente, halla una serie de factores que componen la personalidad caracterizados por:

- Actividad general: ritmo rápido de actividades, energía, vitalidad, actividad constante, productividad, eficacia, gusto por la velocidad, rapidez de acción, entusiasmo y vitalidad.

- Retraimiento: seriedad, lentitud, esfuerzo persistencia y autocontrol.

- Ascendencia: autodefensa, liderazgo, convicción y oratoria.

- Sociabilidad: amistad, contacto social y centro de atención.

- Estabilidad emocional: optimismo, alegría, continuidad en los estados de ánimo e intereses, recato y sentimientos de buena salud.

- Objetividad: imperturbabilidad.

- Amabilidad: tolerancia hacia la acción hostil, aceptación de la dominación y respeto por los otros.

- Actitud pensativa: reflexión, equilibrio mental y observación.

- Relaciones personales: tolerancia hacia las personas y fe en las instituciones.

- Masculinidad: interés por las actividades y vocaciones masculinas, dureza, firmeza ante el temor, inhibición de las manifestaciones emocionales y desinterés en las vestimentas. 
En sexto lugar, el Tridimensional Personality Questionnaire (Cloninger, Thomas y Dragan, 1991) incluye tres dimensiones de temperamento: búsqueda de novedad, evitación del daño y dependencia de recompensa. Sin embargo, posteriormente, Cloninger, Przybeck, Svrakic y Wetzel (1994) amplían su modelo a siete factores, divididos en los de temperamento y los de carácter. En cuanto a los primeros, se encuentran los tres obtenidos en 1991 y otro nuevo: persistencia. Y, respecto a los segundos, aparecen la autodirección, la cooperatividad y la autotrancendencia. Pero, puesto que el instrumento creado inicialmente solamente evalúa tres dimensiones de temperamento, solventan el problema fabricado el Temperament and Character Inventory (Cloninger y otros, 1994).

En último lugar, existen otras teorías acerca de los rasgos de personalidad. Entre ellas puede destacarse la de Pinillos, a partir de la cual crea un cuestionario (Sánchez y Pinillos, 2010) cuyas dimensiones principales son tres: control, extraversión y paranoidismo, añadiendo también dos escalas complementarias: sinceridad y número de dudas. Sin embargo, otros autores sostienen que son dos grandes los rasgos de la personalidad, como Digman (1997), Young, Peterson y Higgins (2001) o Elliot y Thrash (2002). E, incluso, algunos autores (Amigó, 2005; Amigó, Caselles y Micó, 2010) aportan evidencias sobre la existencia de un único rasgo de personalidad.

A modo de resumen, se puede considerar que todavía no hay una teoría de rasgos universalmente aceptada, aunque, como señalan Bermúdez y otros (2011), actualmente la investigación comienza a centrarse en los modelos pentafactoriales de, por un lado, Costa y McCrae (1978) y, por otro, Caprara y otros (1993).

\section{LA INNOVACIÓN EDUCATIVA}

\subsection{Conceptualización}

Al igual que sucede con los conceptos de personalidad y de rasgos, existen diversas definiciones de innovación educativa. Por ejemplo, tras un análisis sobre el término, Monge (2013) propone que la innovación educativa puede entenderse como el conjunto de ideas, actitudes, procesos de cambio y estrategias de indagación, más o menos sistematizados y efectuados de manera colectiva, orientados a generar conocimiento desde la información propia de la organización, cuyo fin es mejorar la práctica educativa, buscando la calidad, y propiciar la disposición a indagar, descubrir, reflexionar y criticar. 
Por otro lado, la innovación educativa puede caracterizarse de una u otra manera según el modelo adoptado (Juárez, 2011):

- Modelo sistémico funcional: entiende la innovación educativa como un proceso de cambio.

- Modelo heurístico: la caracteriza como solución de problemas.

- Modelo generativo: definida como crecimiento personal e institucional.

También, la innovación educativa puede atender a las tres perspectivas comúnmente aceptadas: tecnológica, cultural y sociopolítica (Juárez, 2011).

Por todo ello, este término puede interpretarse desde distintos modelos, perspectivas e ideas de los múltiples autores. Sin embargo, aquí se pretende adoptar una posición integradora al respecto.

\subsection{Tipología}

Rivas (2000) propone una clasificación para los tipos de innovación educativa, atendiendo a:

- Los componentes:

- Las operaciones para el mantenimiento de los límites del sistema.

- El tamaño y extensión.

- Las instalaciones.

- Los tiempos.

- Los objetivos instructivo-formativos.

- La definición de roles.

- Los valores, concepciones y creencias.

- La estructura y las relaciones entre las partes.

- Los métodos de socialización.

- La conexión entre los sistemas.

- Los procedimientos:

- Didácticos.

- Organizativo-curriculares.

- Materiales.

- Estructuración de las clases.

- El modo de realización:

- Adición.

- Reforzamiento.

- Eliminación.

- Sustitución.

- Alteración. 
- Reestructuración.

- La intensidad:

- Marginales.

- Adicionales.

- Fundamentales.

- La amplitud:

- Institucionales.

- Facultativas o parciales.

Posteriormente, Moschen (2008) amplía esta clasificación añadiendo el criterio de los costes (altos, medios y bajos), el del tiempo (largo plazo, medio plazo y corto plazo) y el criterio referido a la participación y gestión institucional (autogestionada, parcialmente asistida y totalmente asistida).

Finalmente, cabe señalar que las innovaciones educativas pueden responder a diversas tipologías y clasificarse en varios criterios. Y esto lo pueden hacer simultáneamente o progresivamente (es decir, al comienzo del proceso de cambio son de una determinada manera y al final del mismo pueden ser significativamente diferentes).

\subsection{Facilitadores y restrictores}

Las innovaciones educativas se encuentran mediadas por diversas variables. De hecho, generalmente, las investigaciones sobre la temática que emplean el estudio de casos suelen presentar una serie de restrictores e impulsores de las innovaciones educativas de cada situación o contexto específico. Y, a partir de ellas, algunos autores proponen unas variables comunes que influyen sobre los procesos de cambio.

Por ejemplo, Torre (2002), a partir de un estudio de casos, agrupa las variables que dificultan la innovación educativa en torno a:

- Los individuos. Aquí pueden identificarse aspectos como el hábito, la primacía, la percepción o retención selectiva, el superego, la falta de seguridad en sí mismos, los sentimientos de amenaza y temor, la ignorancia y el dogmatismoautoritarismo.

- Las organizaciones educativas. En este factor se hallan elementos como la homeostasis, la dependencia, el status quo, los valores y costumbres, las relaciones interpersonales, la satisfacción grupal, la movilidad-inestabilidad de los docentes y la gestión de la innovación. 
- El proceso inductivo. Pueden destacarse los objetivos y fines de la educación, la clasificación de los contenidos, la evaluación, el trabajo a reloj y la desconexión teoría-práctica.

- El sistema educativo. Son de especial importancia la tendencia del sistema a la uniformidad, la centralizacióndescentralización del sistema, la falta de competitividad, el aislamiento y la escasa inversión (tanto en la actualización de los recursos humanos como materiales).

- El sistema social. Se agrupan los valores y estructura social, la madurez del sistema y el tradicionalismo.

Por su parte, Rivas (2000) clasifica los elementos impulsores de la innovación educativa atendiendo a su procedencia:

- Los internamente inducidos:

- La categoría deontológico-vocacional:

- Imperativo ético.

- Vocación docente.

- La preocupación por el otro:

- Aprovechamiento académico.

- Atención a la persona.

- La proyección profesional:

- Valoración profesional.

- Autorrealización docente.

- Los externamente inducidos:

- Los factores extrainstitucionales:

- Prescripciones del sistema.

- Demandas sociales.

- Los factores intrainstitucionales:

- Adaptación al rol institucional.

- Efecto de los recursos materiales.

- Efecto de la tecnología disponible.

Por otro lado, este autor también señala que los factores restrictores de las innovaciones educativas se centran sobre:

- Los valores, normas y estructuras del sistema social.

- La estructura del sistema escolar.

- La propia naturaleza de la educación y las peculiaridades de la tarea docente.

- Los tipos y características de las innovaciones.

Además, tras un análisis cualitativo, este mismo autor identifica otros elementos restrictores de la innovación educativa; tales como: esfuerzo suplementario, respuesta docente inmediata, rigidez del 
sistema educativo, inseguridad y rutinas, limitaciones en la formación pedagógica, falta de apoyo profesional, incomprensión y actitudes inadecuadas, recursos instrumentales, incertidumbre de los resultados y/o relación costes-beneficios.

También, tras un análisis factorial obtiene unos factores impulsores y otros restrictores. Los primeros son: (a) explotación proyectiva optimizante, (b) apertura del rol, (c) respuesta adaptada al rol institucional, (d) vinculación instrumental-contextual de la tarea y (e) búsqueda de estima personal. Y los segundos son: (a) artesanalidad insularizada, (b) disfuncionalidad operativa, (c) costo ostensible y beneficios diluidos, (d) compulsividad del sistema, (e) restricciones instrumentales.

Carbonell (2001) realiza un análisis teórico sobre las variables que influyen sobre la innovación educativa. Así, propone, por un lado, una serie de factores que identifican la innovación educativa $y$, por otra parte, otros que la dificultan (ver Tabla 4).

\begin{tabular}{|c|c|}
\hline 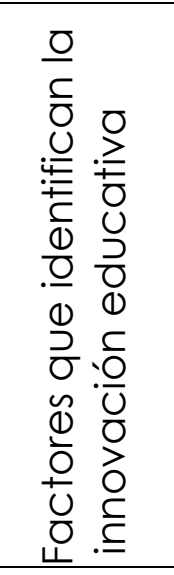 & $\begin{array}{l}\text { Equipos docentes sólidos y comunidad } \\
\text { educativa receptiva } \\
\text { Redes de intercambio y cooperación, } \\
\text { asesores y colaboradores críticos y otros } \\
\text { apoyos externos } \\
\text { Planteamiento de la innovación y el cambio } \\
\text { dentro del contexto territorial } \\
\text { Clima ecológico y rituales simbólicos } \\
\text { Institucionalización de la innovación } \\
\text { La innovación, si no avanza, retrocede } \\
\text { Vivencia, reflexión y evaluación }\end{array}$ \\
\hline 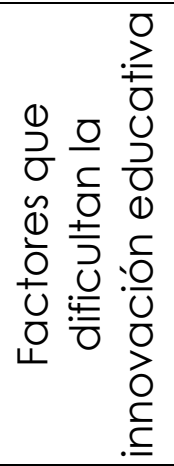 & $\begin{array}{l}\text { Resistencias y rutinas del profesorado } \\
\text { Individualismo y corporativismo interno } \\
\text { Pesimismo y malestar docente } \\
\text { Efectos perversos de las reformas } \\
\text { Paradojas del doble currículo } \\
\text { Saturación y fragmentación de la oferta } \\
\text { pedagógica } \\
\text { Divorcio entre investigación universitaria y } \\
\text { práctica escolar }\end{array}$ \\
\hline
\end{tabular}

Tabla 4. Factores que influyen en las innovaciones educativas (Carbonell, 2001)

Recientemente, una investigación de gran relevancia sobre la innovación educativa en el contexto español es la llevada a cabo por el Ministerio de Educación (2011). En ella, se presentan diversas variables 
que influyen sobre la innovación educativa desde el punto de vista de los distintos agentes del cambio implicados en las mismas. Así, los factores comunes de todos los miembros participantes hacen referencia a los aspectos relacionados con la organización, el profesorado y alumnado, la comunidad educativa $y$, finalmente, la validez del proyecto.

\section{LAS CARACTERÍSTICAS PERSONALES PARA PROMOVER CAMBIOS EN EDUCACIÓN}

Siguiendo con la línea argumental del apartado anterior, las características individuales de los sujetos pueden ser variables que inciden en la innovación educativa.

Pero, además, son varios los autores que sostiene que esta última puede ejercer influencia sobre la personalidad (como Ortega, Ramírez, Torres, López, Servín, Suárez y Ruiz, 2007).

También, existen algunos estudios que muestran cómo se caracterizan los docentes innovadores en cuanto a personalidad se refiere. Respecto a esto, Huberman (1973) realiza una revisión y análisis de las investigaciones previas, donde recoge que:

- Para Harvey (1967)5, los docentes innovadores son personas con un alto grado de abstracción y con una mayor capacidad para apartarse de situaciones inmediatas, menos absolutistas, de mayor relativismo, libres para resolver problemas y buscar soluciones sin temor al castigo por desviarse de la verdad oficial y de los imperativos sociales, con una acentuada orientación práctica, con un comportamiento exploratorio, que aceptan riesgos, etc.

- Para Miles (1964)5, son personas innovadoras que sienten con fuerza la necesidad de autonomía, éxito y orden, así como de ayudar a los demás y de verse apoyadas por otros.

- Para Lazarsfield y Katz (1963)5, son personas que aceptan riesgos, poseen sentido del deber profesional, con confianza en su propia capacidad para controlar el medio...

Investigaciones más recientes al respecto pueden ser las realizadas por Ríos $(2004,2006 a, 2006 b$ y 2009). La estrategia común a todas ellas son las entrevistas en profundidad, que son utilizadas para determinar cómo de innovadores son los docentes. En cambio, fundamentalmente la variable personalidad es hallada mediante

5 Citado/s en Huberman (1973). 
diferentes instrumentos estandarizados (como el Myers-Briggs Type Indicator o el Inventario de Preferencias de Personalidad de Edward). Finalmente, Ríos concluye que los docentes innovadores son personas:

- Persistentes, resistentes, metódicas y planificadoras (2004).

- Con alta motivación pedagógica y social (2006a).

- Realistas, prácticas, no interesadas en lo que no creen que es útil, que las encanta organizar y dirigir actividades, y emplean más el pensamiento para su vida exterior y la sensación de su vida interior (2006b).

- Cercanas y afectuosas, cariñosas y alegres, sobreprotectoras, y consecuentes y honestas (2009).

Posteriormente, Monge (2013) presenta una serie de hipótesis teóricas donde plantea qué rasgos de personalidad poseen los docentes más innovadores. Aunque, si bien es cierto que existen indicios que lo sustentan, todavía no existe evidencia empírica significativa en este contexto. Así, fundamentalmente este autor concluye que:

- Desde la teoría de la personalidad de Cattell (1949)6, se puede esperar que los docentes innovadores puntúen, por un lado, bajos en dominancia (caracterizando a la persona como cooperativa) y en autosuficiencia (integrándose en el grupo) y, por otro lado, puntúen altos en atrevimiento (siendo emprendedores), abstracción (caracterizándose como imaginativos) y, especialmente, abiertos al cambio.

- Según el modelo de los cinco factores de Costa y McCrae $(1978)^{7}$, se puede hipotetizar que los docentes innovadores sean extravertidos (sociables y asertivos), abiertos al cambio (creativos, con amplios intereses y flexibles) y amables (cooperativos y empáticos).

- A partir de la perspectiva de Caprara y sus colaboradores (1993) 7 , los docentes que innovan pueden puntuar alto en afabilidad (siendo cooperativos), tensión (porque son reflexivos) y apertura mental (caracterizándose como cultos, informados y abiertos a experiencias nuevas).

- Desde los planteamientos de Eyseck (1975)7, se puede esperar que los docentes que más innovan en su práctica educativa posean como rasgos primarios: sociabilidad, aventura y racionalidad; y como rasgo secundario: bajo psicoticismo.

- Atendiendo al modelo de Guilford y Zimmerman (Guilford, 1959) ${ }^{7}$, posiblemente los docentes innovadores sean retraídos (persistente en el esfuerzo), sociables (con contacto social),

${ }^{6}$ Citado/s en Monge (2013).

7 Citado/s en Monge (2013). 
con actitud pensativa (reflexivos y observadores, sobre todo de ellos mismos) y puntúen alto en relaciones personales (fe en las instituciones sociales).

- Siguiendo la propuesta de Cloninger y otros (1991 y 1994) $)^{7}$ es probable que los docentes más innovadores correlacionen positivamente en las dimensiones búsqueda de novedades y cooperatividad.

- Según los postulados de Sánchez y Pinillos $(2010)^{7}$ se puede esperar que los docentes que más innovan posean alta extraversión (sociabilidad y facilidad para las relaciones sociales) y bajo paranoidismo (tendencia rígida-flexible).

- Con respecto a algunos modelos bifactoriales (como Digman 1997; Young y otros, 2001)7, es posible que cuanto más innovadores sean los docentes éstos posean, por un lado, mayor factor beta (extraversión, apertura al cambio y crecimiento personal) y, por otro lado, plasticidad (extraversión y apertura a la experiencia).

Por otro lado, existen diversos autores que relacionan las características individuales con la innovación educativa. Así, si bien es cierto que la Psicología es una ciencia básica y auxiliar de los procesos educativos, los gestores del cambio utilizan las diversas especialidades de la psicología. Por una parte, dichos agentes deben motivar al conjunto de la organización para generar cambios y mantenerlos (Carretero, Liesa, Mayoral y Mollà, 2008), por lo que utilizan la Psicología de la motivación. Por otro lado, emplean también la Psicología social dentro del proceso de socialización de los profesores y asesores (Vicente, 2000), así como la psicología de las organizaciones y los grupos, sobre todo enfocado a las cuestiones de liderazgo y roles (Carpio y Guerra, 2008; Yanes y Ries, 2013). Además, utilizan conocimientos sobre Psicología de la emoción (Torrego, 2008). Por otro lado, el proceso de comunicación es elemental (López, 2008), cobrando especial relevancia la Psicología del lenguaje. En cambio, por lo que respecta a la Psicología de la personalidad, las investigaciones hasta el momento tratan de una manera no sistemática esta temática. Entre ellas, se puede concluir que los gestores de la innovación educativa deben demostrar conocimiento sobre el desarrollo evolutivo de la personalidad y ayudar a los miembros de las organizaciones a desarrollar su personalidad (Escudero, Vallejo y Botías, 2008). Además, dentro del contexto del asesoramiento colaborativo, estos agentes del cambio han de partir de las características psicológicas personales, ideas y actitudes de los miembros de las organizaciones (Carpio y Guerra, 2008; Murillo, 2008; Tapia, 2008). 
En síntesis, los rasgos de personalidad y la innovación educativa son dos conceptos relacionados entre sí. Esto se debe a tres motivos fundamentales: (1) a través de ciertas acciones educativas innovadoras se puede desarrollar la personalidad de los estudiantes, (2) los docentes que más innovan en sus prácticas educativas se caracterizan con ciertos rasgos de personalidad y, finalmente, (3) las características individuales están inmersas en la gestión del cambio organizativo.

\section{CONCLUSIÓN}

\section{Marco teórico}

La literatura científica señala que existen diversas variables que influyen sobre la innovación educativa (que la potencian y restringen), pero írealmente los conocimientos sobre los rasgos de personalidad de los agentes del cambio y mejora educativa pueden actuar como facilitadores de la innovación en educación? Al respecto, la respuesta parece ser afirmativa.

En cuanto a la innovación educativa, ésta puede entenderse como el conjunto de ideas, actitudes, procesos de cambio y estrategias de indagación, más o menos sistematizados y efectuados de manera colectiva, orientados a generar conocimiento desde la información propia de la organización, cuyo fin es mejorar la práctica educativa, buscando la calidad, y propiciar la disposición a indagar, descubrir, reflexionar y criticar. Además, puede responder a distintos modelos (sistémico, heurístico y generativo) y diversas perspectivas (tecnológica, cultural y sociopolítica) (Juárez, 2011). También, puede clasificarse atendiendo, por un lado, a los componentes, el modo de realización, la intensidad y la amplitud (Rivas, 2000), y, por otra parte, puede catalogarse según el tiempo, el coste y la participación y gestión individual (Moschen, 2008). En torno a las variables que inciden sobre los procesos innovadores en el ámbito educativo, generalmente se agrupan en aquellas que los facilitan y otras que los dificultan (Ministerio de Educación, 2011; Carbonell, 2001; Rivas, 2000). Sin embargo, ninguno establece de manera consistente los conocimientos que poseen los gestores de la innovación como impulsores de la misma.

Respecto a los rasgos de personalidad, no existe una visión universalmente aceptada, si bien parece que los modelos pentafactoriales de, por un lado, Costa y McCrae (1978) y, por otra parte, de Caprara y otros (1993) comienzan a establecerse como principales perspectivas de la investigación actual (Bermúdez y otros, 2011). Por ello, dependiendo de las ideas de cada autor, se pueden 
establecer varias teorías y modelos. Concretamente, donde más difieren los teóricos es en determinar cuáles y cuántos son los rasgos de personalidad, así como en acordar qué variables influyen en mayor medida para su desarrollo (factores ambientales o factores genéticos). Por ejemplo, Cattell y otros (1949) sostienen que existen 16 rasgos de personalidad y que éstos se encuentran determinados principalmente por causas ambientales. En contra, Eysenck y Eysenck (1975) sostienen que las personas poseen tres rasgos fundamentales de personalidad, estando influidos por variables hereditarias. Sin embargo, también pueden destacarse otras teorías diferentes entre sí; como el modelo de los cinco factores (Caprara y otros, 1993; Costa y McCrea, 1978), el de Gray (1981) o el de Zuckerman (2002), la teoría de Cloninger y otros (1991, 1994), etc. Pero, a pesar de todas estas diferencias, la mayoría de los modelos al respecto señalan que los rasgos de personalidad son los elementos que mejor definen la estructura de la personalidad.

Pero, tras todo ello, ¿̇qué conexión hay entre los rasgos de personalidad y la innovación educativa? La respuesta puede ir dos direcciones. Por un lado, están las investigaciones que determinan la influencia de la innovación sobre la personalidad y, por otro lado, se encuentran aquellas que hallan la determinación de la personalidad sobre la innovación. En el primer caso, puede destacarse la existencia de estudios (como Ortega y otros, 2007) donde, a través de Proyectos de Innovación Educativa, se desarrolla parcialmente la personalidad del alumnado. Y, en el segundo caso, hay varios estudios (Monge, 2013; Ríos, 2004, 2006a, 2006b y 2009; Huberman, 1973) que analizan los rasgos de personalidad de los docentes que más innovan en sus prácticas pedagógicas.

También, algunos estudios señalan que las características personales de los gestores del cambio en los centros educativos son de especial importancia para llevar a la práctica innovaciones en materia educativa. Concretamente, esas características se centran en cuestiones de motivación (Carretero y otros, 2008), de socialización por parte de los profesores y asesores (Vicente, 2000), de liderazgo, roles y necesidades (Carpio y Guerra, 2008; Yanes y Ries, 2013), de emoción (Torrego, 2008), de comunicación (López, 2008) y de personalidad (Carpio y Guerra, 2008; Escudero y otros, 2008; Murillo, 2008; Tapia, 2008). Si bien es cierto que todas estas investigaciones tratan algunas de las características personales, ninguna de ellas trata sistemáticamente los rasgos de personalidad. Precisamente, si estas características son básicas en los procesos de cambio, la personalidad también es fundamental, siendo los rasgos los elementos que mejor explican la estructura de la misma. También, que los gestores de las innovaciones 
educativas conozcan cómo son sus compañeros es un aspecto principal para poder generarlas y mantenerlas.

\section{Propuesta metodológica de investigación}

Por todo ello, se puede hipotetizar que si esos gestores manejan de una manera adecuada sus conocimientos sobre los rasgos de personalidad de sus compañeros, esas habilidades pueden actuar como facilitadores de la innovación educativa. Así, no sólo es necesario tener los conocimientos, sino que hay que saber utilizarlos.

El objetivo perseguido es determinar cómo los diferentes agentes de la innovación educativa utilizan sus conocimientos sobre los rasgos de personalidad de sus compañeros como factores que impulsan el cambio hacia la mejora. Concretamente, el paradigma cualitativo cobra especial relevancia en torno al esclarecimiento de preguntas de investigación con el pronombre "cómo".

Desde una postura cualitativa, la percepción de los participantes en los procesos, en este caso, de cambio educativo cobra especial importancia para la investigación. Un posible diseño metodológico puede ser orientado hacia los grupos de discusión, como técnica de triangulación de informantes, y las entrevistas. La propuesta que aquí se plasma se hace en torno a: (1) un grupo de discusión constituido por expertos en procesos de innovación, donde se pretende analizar cómo ellos perciben que utilizan los conocimientos sobre rasgos de personalidad para gestionar esos cambios; (2) entrevistas en profundidad con el profesorado implicado en las innovaciones en las que intervienen los expertos, con el objetivo de poder contrastar información desde diversas fuentes; (3) un estudio de caso único en un centro educativo para analizar las similitudes entre las percepciones de los participantes y la práctica real. Con todo ello (triangulación de estrategias, informantes y momentos) se espera conseguir una validez considerable.

A modo de conclusión final, puede destacarse que la innovación educativa es un conjunto de procesos y otros elementos novedosos cuyo último objetivo es la mejora del alumnado. Precisamente, los conocimientos que poseen los gestores de las innovaciones educativas, entre otras variables, facilitan la creación y el mantenimiento de éstas. 
Los conocimientos sobre rasgos de personalidad del profesorado como facilitadores de la innovación educativa: estado del arte

\section{REFERENCIAS BIBLIOGRÁFICAS}

AMIGÓ, S. (2005). La teoría del rasgo único de personalidad. Hacia una teoría unificada del cerebro y la conducta. Valencia: Universidad Politécnica de Valencia.

BERMÚEDEZ, J., PÉREZ-GARCÍA, A. M., RUIZ, J. A., SANJUÁN, P. y RUEDA, B. (2011). Psicología de la Personalidad. Madrid: Universidad Nacional de Educación a Distancia.

CAPRARA, G. V., BARBARANELLI, C. y BORGOGNI, L. (1993). Big Five Questionnaire. Florencia: Organizzazioni Seciali.

CARBONELL, J. (2001). La aventura de innovar. El cambio en la escuela. Madrid: Ediciones Morata.

CATELL, R. B., CATELL, A. K. y CATELL, H. E. P. (1949). Sixteen Personality Factor Questionnaire. Champaign: Institute for Personality and Ability Testing.

CLONINGER, C. R., THOMAS, R. P. y DRAGAN, M. S. (1991). The Tridimensional Personality Questionnaire: normative data. Psychological Reports, 69 (3), pp. 1047-1057.

DOI: http://dx.doi.org/10.2466/pr0.1991.69.3.1047

CLONINGER C. R., PRZYBECK T. R., SVRAKIC D. M. y WETZEL R. D. (1994). The Temperament and Character Inventory (TCI): a guide to its development and use. Washington: Center for Psychology of Personality

COSTA, P. T. y MCCRAE, R. R. (1978). Neuroticims, Extraversion and Opennes-Personality Inventory. Florida: Psychological Assesment Resources.

DIGMAN, J. M. (1997). Higher-order factors of the big five. Journal of Personality and Social Psychology, 73, pp. 1246-1256.

DOI: http://dx.doi.org/10.1037/0022-3514.73.6.1246

ELLIOT, A. J. y THRASH, T. M. (2002). Approach-Avoidance motivation in personality: approach and avoidance temperaments and goals. Journal of Personality and Social Psychology, 82, pp. 804-818.

DOI: http://dx.doi.org/10.1037/0022-3514.82.5.804

EYSENCK, H. J. y EYSENCK, S. B. G. (1975). Manual of the Eysenck Personality Questionnaire. Londres: Hodder and Stoughton.

GIL PASCUAL, J. A. (2011). Metodología cuantitativa en educación. Madrid: Universidad Nacional de Educación a Distancia.

GRAY, J. A. (1981). A critique of Eysenck's theory of personality. En H. J. EYSENCK (Ed.), A model of personality (pp. 246-276). Nueva York:

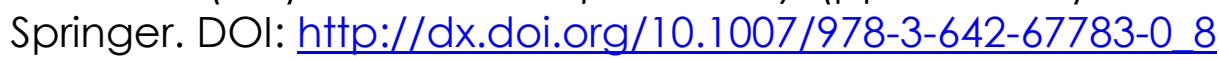

GUILFORD, J. P. (1959). Personality. Nueva York: McGraw-Hill.

HUBERMAN, A. M. (1973). Cómo se realizan los cambios en educación: una contribución al estudio de la innovación. París: UNESCO. 
JUÁREZ, H. M. (2011). Marco teórico, profesional y legal. En Ministerio de Educación, Estudio sobre la innovación educativa en España (pp. 20-56). Madrid: Ministerio de Educación.

MONGE, C. (2013). Los rasgos de personalidad de los docentes innovadores: un estudio teórico. En M. C. Pérez-Llantada, A. López y J. Ibias (Coords.), II Foro de Investigadores Noveles. Madrid: Universidad Nacional de Educación a Distancia.

MONTALVO, D. (2012). Personalidad esquizotípica y límite en población general, universitaria y escolar: el cuestionario SQT (tesis doctoral inédita). Madrid: Universidad Complutense de Madrid.

MOSCHEN, J. C. (2008). Innovación educativa. Decisión y búsqueda permanente ( $2^{\mathrm{a}}$ edición). Buenos Aires: Bonum.

PELECHANO, V. y SERVANDO, M. Á. (2004). Qué es la personalidad. Madrid: Biblioteca Nueva.

PÉREZ-GARCÍA, A. M. y BERMÚDEZ, J. (2011). Introducción al estudio de la personalidad: unidades de análisis. En J. BERMÚEDEZ, A. M. PÉREZGARCÍA, J. A. RUIZ, P. SANJUÁN y B. RUEDA, Psicología de la Personalidad (pp. 25-68). Madrid: Universidad Nacional de Educación a Distancia.

RíOS, D. (2006a). Motivaciones y características de personalidad de profesores que innovan en su práctica pedagógica. En I. MAGAÑA (Comp.), I Jornada de Discusión sobre Proyectos de Investigación (pp. 137-156). Santiago de Chile: Universidad de Santiago de Chile.

RIVAS, M. (2000). Innovación educativa. Teoría, procesos y estrategias. Madrid: Editorial Síntesis.

RUIZ, J. A. (2007). Psicología de la personalidad para psicopedagogos ( $3^{a}$ edición). Madrid: Sanz y Torres.

SÁNCHEZ, M. y PINILLOS, J. L. (2010). CEP. Cuestionario de personalidad (10 edición). Madrid: TEA Ediciones.

TORRE, S. de la (Coord.) (2002). Cómo innovar en los centros educativos. Estudio de casos. Barcelona: Cisspraxis.

YOUNG, C. G., PETERSON, J. B. y HIGGINS, D. M. (2001). Higher-order factors of the big five predict conformity: are the neuroses of health?. Personality and Individual Differences, 33, pp. 533-552.

ZUCKERMAN, M. (2002). Zuckerman-Kuhlman Personality Questionnaire: an alternative five-factorial model. En D. RAAD y M. PERUGINI (Eds.), Big Five Assessment (pp. 377-396). Seatle: Hogrefe and Huber Publishers.

\section{Fuentes electrónicas}

AMIGÓ, S., CASELLES, A. y MICÓ, J. C. (2010). General Factor of Personality Questionnaire: only one factor to understand personality? The Spanish Journal of Psychology, 1 (13). [Consultado 
Los conocimientos sobre rasgos de personalidad del profesorado como facilitadores de la innovación educativa: estado del arte

el 18 de abril de 2013], http://pendientedemigracion.ucm.es/info/psi/docs/journal/v13_nl 2010/art5.pdf.

CARPIO, A. y GUERRA, L. M. (2008). Una experiencia de asesoramiento colaborativo como estrategia de apoyo a los profesores para la implementación de un programa de tutorías universitarias. Profesorado. Revista de Currículum y Formación del Profesorado, 12 (1). [Consultado el 22 de enero de 2013], http://www.ugr.es/ recfpro/rev121COL12.pdf.

CARRETERO, R., LIESA, E., MAYORAL, P. y MOLLÀ, N. (2008). El papel de la motivación de los asesores y profesores en el proceso de asesoramiento. Profesorado. Revista de Currículum y Formación del Profesorado, 12 (1). [Consultado el 22 de enero de 2013], http://www.ugr.es/ recfpro/rev121COL4.pdf.

ESCRIVÁ, P. (2009). Estudio psicométrico para la adaptación del cuestionario de personalidad NEO-PI-R a población adolescente española: el JS NEO (tesis doctoral). Castellón: Universidad Jaime I. [Consultado el 18 de abril de 2013], http://www.tdx.cat/bitstream/handle/10803/10514/escriva.pdf;jses sionid=9C1D76B91 E09C3570F21E20D88C4CF01.tdx2? sequence $=1$.

ESCUDERO, J. M., VALLEJO, M. y BOTíAS, F. (2008). El asesoramiento en educación: ¿̇podrían ser las competencias profesionales una contribución positiva? Profesorado. Revista de Currículum y Formación del Profesorado, 12 (1). [Consultado el 22 de enero de 2013], http://www.ugr.es/ recfpro/rev121ART2.pdf.

IMBERNÓN, F. (2006). Actualidad y nuevos retos de la formación permanente. Revista Electrónica de Investigación Educativa, 8 (2). [Consultado el 11 de marzo de 2013], http://redie.uabc.mx/vol8no2/contenido-imbernon.html.

LÓPEZ, J. (2008). Construir la relación de asesoramiento. Un enfoque institucional basado en la comunicación. Profesorado. Revista de Currículum y Formación del Profesorado, 12 (1). [Consultado el 22 de marzo de 2013], http://www.ugr.es/ recfpro/rev121ART12.pdf.

MURILLO, P. (2008). ¿Ayuda u obstáculo? Sentido y credibilidad de la función asesora. Profesorado. Revista de Currículum y Formación del Profesorado, 12 (1). [Consultado el 22 de enero de 2013], http://www.ugr.es/ recfpro/rev121ART9.pdf.

ORTEGA, P., RAMÍREZ, M. E., TORRES, J. L., LÓPEZ, A. E., SERVÍN, C. Y., SUÁREZ, L. y RUIZ, B. (2007). Modelo de innovación educativa. Un marco para la formación y el desarrollo de una cultura de la innovación. Revista Iberoamericana de Educación a Distancia, 10 (1), pp. 145-173. [Consultado el 22 de abril de 2013], http://www.utpl.edu.ec/ried/images/pdfs/modelodeinnovacion.p df. 
RíOS, D. (2004). Rasgos de personalidad de profesores innovadores: autonomía, persistencia y orden. Revista Latinoamericana de Estudios Educativos, 34 (2), pp. 95-112. [Consultado el 21 de abril de 2013], http://redalyc.uaemex.mx/pdf/270/27034205.pdf.

RíOS, D. (2006b). Tipos psicológicos de profesores primarios innovadores: extroversión, sensación, pensamiento y juicio. Revista Latinoamericana de Estudios Educativos, 36 (3), pp. 103-128. [Consultado el 21 de abril de 2013], http://redalyc.uaemex.mx/pdf/270/27036406.pdf.

RíOS, D. (2009). Características personales y profesionales de profesores innovadores. Revista Latinoamericana de Estudios Educativos, 39 (2), pp. 153-169. [Consultado el 21 de abril de 2013], http://www.cee.edu.mx/revista/r2001_2010/r_texto/t_2009_12_07.pdf.

TAPIA, G. (2008). Formación para el asesoramiento a las escuelas: un proceso emergente en México. Profesorado. Revista de Currículum y Formación del Profesorado, 12 (1). [Consultado el 22 de enero de 2013], http://www.ugr.es/ recfpro/rev121COL\&.pdf.

TORREGO, J. C. (2008). Un estudio sobre la utilización de la metodología de procesos como estrategia de formación del profesorado en relación con la mejora de la convivencia. Profesorado. Revista de Currículum y Formación del Profesorado, 1 (12). [Consultado el 22 de marzo de 2013], http://www.urg.es/recfpro/Rev121.html.

VICENTE, M. (2000). Panorama de la formación y el asesoramiento en educación: algunas claves para saber dónde vamos. Profesorado. Revista de Currículum y Formación del Profesorado, 4 (2). [Consultado el 22 de enero de 2013], http://www.ugr.es/ recfpro/rev42ART1.pdf.

YANES, C. y RIES, F. (2013). Liderando el cambio: estudio sobre las necesidades formativas de los futuros docentes de secundaria. Revista Fuentes, 14 (1), pp. 105-124. [Consultado el 03 de abril de 2014], http://institucional.us.es/fuentes/gestor/apartados_revista/pdf/mo nografico/dtmkvxzb.pdf. 
Los conocimientos sobre rasgos de personalidad del profesorado como facilitadores de la innovación educativa: estado del arte

\section{Sobre los autores:}

\section{Carlos Monge López}

Carlos Monge López es docłorando en Planificación e innovación educativo y Profesor Universitario en Formación en el Departamento de Ciencias de la Educación de la Universidad de Alcalá. Forma parte del grupo de investigación "Inclusión y Mejora Educativa: Convivencia y Aprendizaje Cooperativo" en la misma institución. Sus principales líneas de investigación se centran sobre los procesos de cambio educativo, las características del profesorado y el asesoramiento colaborativo.

\section{David Montalvo Saborido}

David Montalvo Saborido es doctor en Psicología evolutiva y de la educación por la Universidad Complutense de Madrid y Profesor Asociado en el Departamento de Ciencias de la Educación de la Universidad de Alcalá. Forma parte del grupo de investigación "Inclusión y Mejora Educativa: Convivencia y Aprendizaje Cooperativo" en la misma institución. Sus principales líneas de investigación se centran sobre los rasgos de personalidad y el asesoramiento para el cambio educativo.

\section{Patricia Gómez Hernández}

Patricia Gómez Hernández es doctoranda en Comunicación, educación y sociedad en la Universidad de Alcalá. Forma parte del grupo de investigación "Aprendiz.es" en la misma institución. Sus principales líneas de investigación se centran sobre las innovaciones educativas mediadas por las Tecnologías de la Información y Comunicación.

\section{Para citar este artículo:}

Monge,C., Montalvo, D. y Gómez, P. (2015). Los conocimientos sobre rasgos de personalidad del profesorado como facilitadores de la innovación educativa: estado del arte. Revista Fuentes, 16, Junio, pp. 173-198. [Fecha de consulta: dd/mm/aaaa].

http://www.revistafuentes.es/

D.O.I.: http://dx.doi.org/10.12795/revistafuentes.2015.i16.08 